\author{
Luc Biedermann $^{a}$ \\ Gerhard Rogler ${ }^{a}$ \\ Jonas Zeitz ${ }^{\mathrm{a}}$
}

\title{
«Mikrobiota-Tuning» en vogue: Von der Ernährung über Probiotika bis zur fäkalen Mikrobiota-Transplantation
}

biologisch eigentlich als Gegenspieler zur Fauna auf die Pflanzenwelt), ist es weiterhin angebracht, sich folgenden begrifflichen Unterschied zu vergegenwärtigen: Während der Begriff Mikrobiota die Gesamtheit aller Mikroorganismen in einem bestimmten Habitat bezeichnet, bezieht sich der Term Mikrobiom auf die Summe der genetischen Information dieses mikrobiellen Konglomerats.

\section{Die intestinale Mikrobiota des Menschen}

Die intestinale Mikrobiota wird fälschlicherweise oft auf die Summe der Darmbakterien reduziert. Zwar machen diese den Löwenanteil der Mikroorganismen aus, allerdings sind in unserem Gastrointestinalsystem, insbesondere dem Dickdarm, physiologisch auch Pilze, Viren und sogenannte Archaeen (alte einzellige Lebewesen ohne Zellorganellen, die wie die Bakterien den Prokaryonten zugerechnet werden) enthalten [1]. Die Zahl der Mikroorganismen in jedem Menschen ist mit geschätzt etwa 100 Billionen $\left(10^{14}\right)$ nahezu unvorstellbar hoch und beinhaltet zwischen 1 und $2 \mathrm{~kg}$ Biomasse [2] bzw. mindestens 300-1000 verschiedene Spezies [2,3]. Die genaue Zahl der verschiedenen "Arten» ist hierbei schwierig zu bestimmen und hängt letztlich von einer bioinformatischen Konvention ab. In der Regel wird eine mindestens $97 \%$ ige SequenzHomologie als Schwelle für die Zugehörigkeit zu einer «Spezies» bzw. soge- nannte «operationale taxonomische Einheit» (OTU) angesehen [4].

Der grösste Wissenszuwachs hat sich in den letzten Jahrzehnten durch einen Sprung weg von der Kultur (nur geschätzt 10\% aller Mikroorganismen sind überhaupt kultivier- und damit nachweisbar) hin zu den hochautomatisierten Sequenzierapparaten ergeben, die in der Lage sind, eine riesige Zahl von Sequenzen pro Zeit zu analysieren.

Bakterien machen - wie bereits erwähnt - den Grossteil der Mikroorganismen der intestinalen Mikrobiota aus, wobei hier wiederum vier Stämme, sogenannte Phyla, überwiegen: Firmicutes und Bacteroidetes (zusammen etwa 90\% [5]) sowie Actinobacteria und Proteobacteria. Ausgehend von dieser Einteilung erfolgt die für die Biologie typische weitere Subklassifizierung, was sich am Beispiel des Bakteriums Escherichia coli wie folgt ergibt: Domäne (Domain): Bakterien; Reich (Kingdom): Eubakterien; Abteilung/Stamm (Phylum): Proteobacteria; Klasse (Class): Gammaproteobacteria; Ordnung (Order): Enterobacteriales; Familie (Family): Enterobacteriaceae; Gattung (Genus): Escherichia; Art (Species): Escherichia coli.

All diesen Erkenntnissen zum Trotz stehen wir beispielsweise noch immer am Anfang eines besseren Verständnisses davon, wie Störungen der mikrobiellen Zusammensetzung eine Rolle bei der Pathogenese verschiedener Erkrankungen spielen. Zunächst müsste Einigkeit darüber herrschen, was genau unter einer normalen hu- 
manen intestinalen Mikrobiota-Komposition zu verstehen ist. Auf den ersten Blick erscheint dies relativ einfach durch übliche mathematische Definitionen wie Mittelwert, Verteilungsmuster und Standardabweichung $\mathrm{zu}$ bewerkstelligen, jedoch ist dies keineswegs der Fall. Noch immer gehen grosse Konsortien dieser Fragestellung nach [6]. Dies liegt insbesondere daran, dass die normale Zusammensetzung der humanen Mikrobiota sowohl intestinal als auch in anderen Körperregionen extrem variabel ist. So zeigten etwa Untersuchungen an einer grossen Population gesunder Erwachsener, dass es hinsichtlich der Zusammensetzung auf Phyla-Ebene Individuen mit über 90\% Bacteroidetes-Anteil gibt, wohingegen am anderen Ende des Spektrums solche mit einer ähnlich hohen Fraktion von Firmicutes stehen [7]. Dazwischen gibt es unzählige Abstufungen dieser beiden Extreme allein auf diese beiden Phyla bezogen - und sämtliche Spielmuster der Zusammensetzung entsprechen einer normalen Mikrobiota. Beide Gruppen sind gesund und zeigen keine Hinweise auf Fehlfunktionen der Verdauung. Dieser Sachverhalt kann kaum genug betont werden, da es immer wieder Patienten gibt, die sich in diversen kommerziellen Labors (semi-)quantitativen Bestimmungen spezifischer Mikroben im Stuhl unterziehen. Dies, obwohl die quantitative Interpretation einer allfälligen spezifischen Störung in der Zusammensetzung sowie auch die teilweise kombiniert erwähnten Empfehlungen für entsprechende Massnahmen zu potenziellen Korrekturbemühungen der Mikrobiota aktuell jeder wissenschaftlichen Grundlage entbehren.

So sehr die interindividuelle mikrobielle Zusammensetzung auch bei gesunden Menschen aus dem gleichen Kulturkreis variieren kann, so erstaunlich stabil hat sich die intraindividuelle Zusammensetzung der Mikrobiota bei den einzelnen Individuen gezeigt [8]. Bisher wissen wir nur wenig darüber, wie diese komplexe Komposition über die Zeit in ihrer Zusammensetzung moduliert wird, welche Einflüsse exogene Faktoren (Diät, Medikamente, Reisen, Temperatur, akute und chronische Erkrankungen, externe $\mathrm{Pa}$ thogene usw.) kurz- und längerfristig ausüben und wie vor allem diese hohe relative Stabilität über viele Jahre aufrechterhalten wird. Ohne Zweifel übt z.B. die Diät einen Effekt auf die mikrobielle Zusammensetzung aus [9], wie auch in verschiedenen Studien beim Menschen [10] (und auch im Tierversuch mit Mäusen [11]) mit Adipositas gezeigt werden konnte. Spezifische Nahrungsbestandteile und deren Metaboliten könnten durch ihre Veränderung der mikrobiellen Zusammensetzung einen wichtigen Einfluss auf verschiedene Erkrankungen nehmen, was z.B. durch Studien mit L-Carnitin, einem Bestandteil von rotem Fleisch, in Mäusen mit einem erhöhten Atheroskleroserisiko gezeigt wurde [12]. Wenngleich also der Einfluss der Ernährung auf die Zusammensetzung unserer intestinalen Mikrobiota ausser Frage steht [13], fehlt aktuell die wissenschaftliche Grundlage, um bestimmte Diäten zur gezielten Manipulation der Mikrobiota in eine wünschenswerte Richtung zu empfehlen.

\section{Probiotika: \\ Aktuelle Bedeutung}

Als Probiotika werden lebende, nicht pathogene Nahrungsbestandteile bezeichnet, welche die intestinale Mikrobiota vorübergehend und mit dem Ziel eines gesundheitsfördernden Effekts beeinflussen. Demgegenüber bezeichnen Präbiotika unverdauliche Polysaccharide, welche als Substrat für Teile der intestinalen Mikrobiota dienen. Präbiotika werden dann zu Oligosacchariden im Darm gespalten und bakteriell fermentiert, wie etwa zu kurzkettigen Fettsäuren, welche wiederum ein Nahrungssubstrat für die Darmschleimhaut darstellen. Eines der bekanntesten und am häufigsten in der Medizin angewendeten Präbio- tika ist wohl die Lactulose bzw. Lactitol für die Behandlung/Prophylaxe der hepatischen Enzephalopathie bei Patienten mit einer Leberzirrhose. Schliesslich ist noch der Begriff Synbiotika, eine Kombination aus Probiotikum und einem für das Probiotikum als Substrat dienende Präbiotikum, gebräuchlich.

Der Wirkmechanismus der Probiotika ist komplex und bis heute nur unvollständig verstanden. Neben einer direkten kompetitiven Wirkung dieser nützlichen Keime auf Pathogene spielen neuromodulatorische Effekte (Reduktion viszerale Hypersensitivität, Wirkung auf enterische Opioid- und Cannabinoidrezeptoren), Unterstützung bei der epithelialen Homöostase und Digestion von für den Menschen unverdaubaren Nahrungsbestandteilen eine wichtige Rolle [14].

Ein erster Fallbericht geht auf Alfred Nissle zurück, welcher 1917 mitten im Krieg bei der Versorgung der Truppen im Lazarett die Beobachtung machte, dass einige wenige Rekruten einen natürlichen Schutz vor den grassierenden und mitunter zum Tode führenden Enteropathogenen zu haben schienen. Kurzum applizierte er an Darminfektionen erkrankten Soldaten Stuhlbestandteile nicht erkrankter, «immuner» Kumpanen, mit beachtlichem Erfolg. Heute wissen wir, dass dieser Effekt auf eine spezifische apathogene Subspezies von E. coli zurückzuführen ist (E. coli Nissle) - eines der aktuell am häufigsten verwendeten Probiotika, unter anderem für die Remissionserhaltung bei milden bis mittelschweren Fällen einer Colitis ulcerosa [15]. In der Schweiz sind seit einigen Jahren verschiedene weitere Präparationen von Keimen erhältlich und für spezifische Indikationen zugelassen, wie z.B. Enterococcus faecium SF68 bei Enteritis und Enterocolitis bei Kindern und Erwachsenen und Saccharomyces boulardii zur Behandlung/Prophylaxe der Antibiotika-assoziierten Diarrhö oder Reduktion der Rezidivgefahr einer Infektion mit Clostridium difficile. Es muss an dieser 
Stelle betont werden, dass die relativ gute Studienlage für Probiotika bei diesen Indikationen im klaren Gegensatz zu all den erhältlichen probiotischen Produkten im Supermarkt und Reformhaus steht. Lebensmittel, die mit probiotischen Keimen angereichert sind, wurden praktisch nie systematisch auf ihren potenziellen Nutzen hin untersucht. Schwerwiegender noch als die fehlende wissenschaftliche Evidenz etwa von probiotischen Joghurts ist sicher die Tatsache zu werten, dass eine grosse Anzahl der zugesetzten Keime den Weg aus der Fabrik-Supermarkt-Kühlkette in den heimischen Kühlschrank nicht überleben [16].

Für Probiotika besteht zum Teil eine hohe Erwartungshaltung, welche im klinischen Alltag nicht immer erfüllt werden konnte. In den letzten Jahren haben sich interessante neue potenzielle Anwendungsgebiete aufgezeigt, und ein Nutzen konnte in methodisch hochwertigen Studien belegt werden. So zeigte sich etwa ein positiver Effekt von Probiotika in der Primärprophylaxe einer hepatischen Enzephalopathie $[17,18]$ bei Patienten mit Leberzirrhose.

Auch konnten verschiedene Studien einen Effekt von Probiotika bei chronischer bzw. rezidivierender Pouchitis zeigen [19, 20], also einer Entzündung des chirurgisch erstellten Enddarmabschnitts aus Ileum-Anteilen nach einer totalen Kolektomie (z.B. wegen therapierefraktärer Colitis ulcerosa). Auch scheinen Probiotika ein Potenzial zur Reduktion von erneuten Diverticulitis-Episoden zu haben [21].

Zweifelsohne hochinteressant sind auch erste Studien, welche einen positiven Effekt von Probiotika auf depressive Symptome beim Menschen nahelegen [22]. In diesem Feld dürften in den nächsten Jahren zahlreiche interessante Forschungsergebnisse zu erwarten sein, schliesslich wurde eine Dysbiose der intestinalen Mikrobiota bei psychiatrischen Erkrankungen, insbesondere den affektiven Störungen [23], bereits wiederholt beschrie- ben [24]. Andererseits erfolgte auch vor 4 Jahren erstmals in einer kontrollierten, verblindeten Studie der direkte Beweis, dass eine mikrobielle Manipulation mithilfe von Probiotika die Gefühlswahrnehmung und Hirnaktivität zu beeinflussen vermag [25].

\section{Fäkale Mikrobiota-}

Transplantation - (zu) rasanter Aufstieg von der Persona non grata zum therapeutischen Panaceum?

Die rezidivierende C.-difficile-Infektion (rCDI) stellt eine schwierige therapeutische und teils auch diagnostische Herausforderung dar. Zwar ist das Ansprechen auf eine antibiotische Therapie mit rund $80 \%$ recht hoch, aber die Minderheit, welche nach einer erfolgreichen Therapie ein Rezidiv erleidet, unterliegt einem deutlich höheren Risiko eines erneuten Rezidivs, was sich wiederum mit jedem konsekutiven Rezidiv mehr oder weniger linear fortsetzt [26]. Seit Langem gilt Metronidazol als Erstlinientherapie, zumindest bei milder bis moderater Form der Erkrankung, wohingegen Vancomycin bei initial schwerem Verlauf z.B. bei hospitalisierten Patienten oder eben bei Rezidiven zum Einsatz kommt [27]. Wie bereits erwähnt, kann die Zugabe von Probiotika das Rezidivrisiko senken [28]. Weitere Therapieoptionen sind in den letzten Jahren hinzugekommen, so etwa die Antibiotika Fidaxomicin und Rifaximin sowie demnächst auch monoklonale Immunoglobuline gegen das Toxin B von C. difficile [29].

Ein neuer Therapieansatz der rCDI ist die fäkale Mikrobiota-Transplantation (FMT). Der Begriff «Stuhltransplantation» für die FMT ist unpräzise und sollte vermieden werden, da nicht der komplette Stuhl, sondern eine mikrobielle Lösung in Form eines Filtrates appliziert wird. Interessanterweise zeigen sich Patienten gegenüber dieser Therapieoption deutlich offener, als man dies erwarten würde, und zum
Teil auch deutlich offener als immer noch viele Ärzte, besonders auch für Indikationen abseits der rCDI [30,31].

Das Prinzip der FMT taucht bereits seit Jahrhunderten unter dem Begriff Transfaunation in der human- bzw. veterinärmedizinischen Literatur auf. Die intestinale Dysbiose, etwa als Resultat von Antibiotikagebrauch, ist eine der wichtigsten Voraussetzungen für eine insuffizient gehemmte Proliferation von C. difficile und damit klinischen Rezidiven durch residuelle Keime. Genau hier setzt das Prinzip der FMT an. Diese adressiert nämlich nicht direkt das Pathogen, sondern stellt eine kausale Behandlung der Dysbiose dar und führt daher zum Wiedererlangen einer «normalen» (wenngleich von der ursprünglichen Zusammensetzung unterschiedlichen) mikrobiellen Zusammensetzung, was in den meisten Fällen das Problem der rCDI löst. Die Erfolgsraten der FMT sind mit über $90 \%$ beeindruckend hoch, was in einer kontrollierten und randomisierten Studie von FMT versus einer alleinigen antibiotischen Therapie mit Vancomycin aus den Niederlanden gezeigt werden konnte. Angesichts der hohen Erfolgsraten wurde sogar ein vorzeitiger Stopp der Studie durch die Ethikkommission auferlegt, da ein Weiterführen für die Patienten, welche nicht in die FMT-Gruppe randomisiert worden wären, in Anbetracht der erdrückend geringeren Erfolgschancen nur durch Antibiotika als unethisch eingestuft wurde [32].

Die zunehmenden Erfahrungen und steigenden Patientenzahlen weltweit sowie die zahlreichen neueren Untersuchungen $\mathrm{zu}$ diesem Thema sollten nicht darüber hinwegtäuschen, dass weiterhin sehr viele offene Fragen betreffend der FMT bestehen, die es in den nächsten Jahren zu beantworten gilt. Weiterhin ist etwa nicht geklärt, welche Art der Applikation («obere gastrointestinale Route» via Duodenal- oder Magensonde vs. Einlauf oder Applikation im Rahmen einer Koloskopie - in den letzten Jahren verdich- 
ten sich die Hinweise, dass letztgenannte Art die effizienteste, aber auch aufwendigste ist) zu bevorzugen ist. Auch ist nicht geklärt, welche Trägerlösung ideal ist, und es existieren widersprüchliche Argumente betreffend der idealen Selektion des Spenders (Donor). Für einen fremden bzw. nicht im selben Haushalt lebenden Donor spricht, dass eine möglichst unterschiedliche mikrobielle Zusammensetzung wünschenswert ist und somit die Chance einer Kolonisation der Spendermikrobiota am höchsten ist. Für einen Spender aus demselben Haushalt mit/ohne genetische Verwandtschaft spricht eine möglichst ähnliche Zusammensetzung der Mikrobiota, die möglicherweise ideal für ein besseres «engraftment» und weniger Gefahr unerwünschter Langzeitfolgen durch die iatrogene mikrobielle Alteration ist.

Wenngleich bis heute keine relevanten bzw. schweren Komplikationen durch die FMT dokumentiert wurden (kurzzeitige fieberhafte Zustände oder Diarrhö/Flatulenz können mitunter auftreten), sollte nicht unerwähnt bleiben, dass durch die mikrobielle Transplantation folgende potenzielle Gefahren bestehen: Einerseits könnte ein infektiöses Agens, eventuell auch ein bisher unbekanntes (man denke hier etwa an die Übertragung z.B. durch Bluttransfusionen von Hepatitis-C-Viren oder Humane Immunde-
fizienz-Viren (HIV) zu einem Zeitpunkt, als diese noch gar nicht bekannt waren), übertragen werden, welches der routinemässigen Testung für die wichtigsten Enteropathogene sowie Hepatitis B/C und HIV natürlich entgehen würde. Andererseits könnte durch die akute und ausgeprägte Alteration der intestinalen Mikrobiota im Empfänger auch auf längere Sicht die Suszeptibilität für Erkrankungen erhöht werden, die mit Störungen der intestinalen Mikrobiota assoziiert sind.

Mag dies bei Erkrankungen wie dem Reizdarmsyndrom oder auch der Adipositas - zu letzterer gibt es einen Fallbericht einer De-novo-Adipositas nach FMT [33], wobei allerdings schwer zwischen einer Gewichtszunahme durch das Überstehen der rCDI und einer «echten» Adipositas zu unterscheiden ist - eher unbedeutend erscheinen, wäre dies bei anderen Entitäten, wo eine intestinale Dysbiose als wesentliches pathogenetisches Element diskutiert wird, von ungleich höherer Relevanz, wie z.B. bei nichtalkoholischer Fettlebererkrankung [34], Kolonkarzinom [35], multipler Sklerose [36] oder der Arteriosklerose [37].

\section{Fazit und Ausblick}

Die FMT stellt eine tiefgreifende und zugleich vollkommen ungerichte- te Möglichkeit einer Manipulation der intestinalen Mikrobiota dar. Für andere Indikationen als die rCDI besteht bis anhin noch keine ausreichende wissenschaftliche Evidenz. Am Vielversprechendsten sind momentan erste Resultate bei der Colitis ulcerosa, wenngleich bisher nicht verstanden ist, warum nur manche Patienten profitieren und warum spezifische Donoren mit deutlich höheren Erfolgsraten assoziiert zu sein scheinen [38-40].

Möglicherweise werden zukünftig mikrobielle Präparationen aus spezifischen Donorbanken bzw. kommerziellen Einrichtungen eine therapeutische Rolle spielen, auch für Indikationen abseits der rCDI. Des Weiteren ist sogar denkbar, dass in einigen Jahren bzw. Jahrzehnten die mikrobielle Komposition beim Akzeptor in spe analysiert und in Abhängigkeit vom Krankheitsbild und dem therapeutischen Ziel massgeschneiderte mikrobielle Präparationen angefertigt werden können. Ob dies jemals möglich sein wird, erscheint zum jetzigen Zeitpunkt zumindest fraglich. Klar ist demgegenüber, dass noch sehr viel weiterführende Forschung notwendig ist, um die Komplexität der Komposition der intestinalen Mikrobiota und deren metabolische und immunologische Funktionen besser zu verstehen, bevor weitergehende therapeutische Entwicklungen überhaupt erst ins Auge gefasst werden können.

\section{Literatur}

1 Hollister EB, Gao C, Versalovic J: Compositional and functional features of the gastrointestinal microbiome and their effects on human health. Gastroenterology 2014;146:1449-1458.

2 Savage DC: Microbial ecology of the gastrointestinal tract. Annu Rev Microbiol 1977;31: 107-133.

3 Eckburg PB, Bik EM, Bernstein CN, Purdom E, Dethlefsen L, Sargent M, Gill SR, Nelson KE, Relman DA: Diversity of the human intestinal microbial flora. Science 2005;308:16351638.

4 Lozupone CA, Knight R: Species divergence and the measurement of microbial diversity. FEMS Microbiol Rev 2008;32:557-578.
5 Turnbaugh PJ, Ley RE, Hamady M, Fraser-Liggett CM, Knight R, Gordon JI: The human microbiome project. Nature 2007;449:804-810.

6 Falony G, Joossens M, Vieira-Silva S, et al: Population-level analysis of gut microbiome variation. Science 2016;352:560-564.

7 Human Microbiome Project Consortium: Structure, function and diversity of the healthy human microbiome. Nature 2012;486:207-214.

8 Faith JJ, Guruge JL, Charbonneau M, Subramanian S, Seedorf $\mathrm{H}$, Goodman AL, Clemente JC, Knight R, Heath AC, Leibel RL, Rosenbaum M, Gordon JI: The long-term stability of the human gut microbiota. Science 2013;341: 1237439 .
9 Albenberg LG, Wu GD: Diet and the intestinal microbiome: associations, functions, and implications for health and disease. Gastroenterology 2014;146:1564-1572.

10 Ley RE, Turnbaugh PJ, Klein S, Gordon JI: Microbial ecology: human gut microbes associated with obesity. Nature 2006;444:1022-1023.

11 Turnbaugh PJ, Bäckhed F, Fulton L, Gordon JI: Diet-induced obesity is linked to marked but reversible alterations in the mouse distal gut microbiome. Cell Host Microbe 2008;3: 213-223. 
12 Koeth RA, Wang Z, Levison BS, et al: Intestinal microbiota metabolism of L-carnitine, a nutrient in red meat, promotes atherosclerosis. Nat Med 2013;19:576-585.

$13 \mathrm{Wu}$ GD, Chen J, Hoffmann C, Bittinger K, Chen Y-Y, Keilbaugh SA, Bewtra M, Knights D, Walters WA, Knight R, Sinha R, Gilroy E, Gupta K, Baldassano R, Nessel L, Li H, Bushman FD, Lewis JD: Linking long-term dietary patterns with gut microbial enterotypes. Science 2011;334:105-108.

14 Ciorba MA: A gastroenterologist's guide to probiotics. Clin Gastroenterol Hepatol 2012; 10:960-968.

15 Kruis W, Fric P, Pokrotnieks J, Lukás M, Fixa B, Kascák M, Kamm MA, Weismueller J, Beglinger C, Stolte M, Wolff C, Schulze J: Maintaining remission of ulcerative colitis with the probiotic Escherichia coli Nissle 1917 is as effective as with standard mesalazine. Gut 2004;53:1617-1623.

16 Scharl M, Geisel S, Vavricka SR, Rogler G: Dying in yoghurt: the number of living bacteria in probiotic yoghurt decreases under exposure to room temperature. Digestion 2011;83: 13-17.

17 Dhiman RK, Rana B, Agrawal S, Garg A, Chopra M, Thumburu KK, Khattri A, Malhotra S, Duseja A, Chawla YK: Probiotic VSL\#3 reduces liver disease severity and hospitalization in patients with cirrhosis: a randomized, controlled trial. Gastroenterology 2014;147:13271337.e3.

18 Lunia MK, Sharma BC, Sharma P, Sachdeva S, Srivastava S: Probiotics prevent hepatic encephalopathy in patients with cirrhosis: a randomized controlled trial. Clin Gastroenterol Hepatol 2014;12:1003-1008.e1.

19 Guslandi M: Role of probiotics in Crohn's disease and in pouchitis. J Clin Gastroenterol 2015;49(suppl 1):S46-49.

20 Mimura T, Rizzello F, Helwig U, Poggioli G, Schreiber S, Talbot IC, Nicholls RJ, Gionchetti P, Campieri M, Kamm MA: Once daily high dose probiotic therapy (VSL\#3) for maintaining remission in recurrent or refractory pouchitis. Gut 2004;53:108-114

21 Tursi A, Brandimarte G, Elisei W, et al: Randomised clinical trial: mesalazine and/or probiotics in maintaining remission of symptomatic uncomplicated diverticular disease - a double-blind, randomised, placebo-controlled study. Aliment Pharmacol Ther 2013;38:741751.
22 Steenbergen L, Sellaro R, van Hemert S, Bosch JA, Colzato LS: A randomized controlled trial to test the effect of multispecies probiotics on cognitive reactivity to sad mood. Brain Behav Immun 2015;48:258-264.

23 Jiang $\mathrm{H}$, Ling Z, Zhang Y, Mao H, Ma Z, Yin Y, Wang W, Tang W, Tan Z, Shi J, Li L, Ruan B: Altered fecal microbiota composition in patients with major depressive disorder. Brain Behav Immun 2015;48:186-194.

24 Cryan JF, Dinan TG: Mind-altering microorganisms: the impact of the gut microbiota on brain and behaviour. Nat Rev Neurosci 2012; 13:701-712.

25 Tillisch K, Labus J, Kilpatrick L, Jiang Z, Stains J, Ebrat B, Guyonnet D, Legrain-Raspaud S, Trotin B, Naliboff B, Mayer EA: Consumption of fermented milk product with probiotic modulates brain activity. Gastroenterology 2013; 144:1394-1401.

26 Kelly CP, LaMont JT: Clostridium difficile more difficult than ever. N Engl J Med 2008; 359:1932-1940.

27 Debast SB, Bauer MP, Kuijper EJ; European Society of Clinical Microbiology and Infectious Diseases: European Society of Clinical Microbiology and Infectious Diseases: Update of the treatment guidance document for Clostridium difficile infection. Clin Microbiol Infect 2014;20:1-26.

28 Johnston BC, Ma SSY, Goldenberg JZ, Thorlund K, Vandvik PO, Loeb M, Guyatt GH: Probiotics for the prevention of Clostridium difficile-associated diarrhea: a systematic review and meta-analysis. Ann Intern Med 2012; 157:878-888.

29 Wilcox MH, Gerding DN, Poxton IR, et al: Bezlotoxumab for prevention of recurrent Clostridium difficile infection. N Engl J Med 2017;376:305-317.

30 Kahn SA, Gorawara-Bhat R, Rubin DT: Fecal bacteriotherapy for ulcerative colitis: patients are ready, are we? Inflamm Bowel Dis 2012;18: 676-684.

31 Zeitz J, Bissig M, Barthel C, Biedermann L, Scharl S, Pohl D, Frei P, Vavricka SR, Fried M, Rogler G, Scharl M: Patients' views on fecal microbiota transplantation: an acceptable therapeutic option in inflammatory bowel disease? Eur J Gastroenterol Hepatol 2017;29: 322-330.
32 van Nood E, Vrieze A, Nieuwdorp M, Fuentes S, Zoetendal EG, de Vos WM, Visser CE, Kuijper EJ, Bartelsman JF, Tijssen JG, Speelman P, Dijkgraaf MG, Keller JJ: Duodenal infusion of donor feces for recurrent Clostridium difficile. N Engl J Med 2013;368:407-415.

33 Alang N, Kelly CR: Weight gain after fecal microbiota transplantation. Open Forum Infect Dis 2015;2:ofv004.

34 Mouzaki M, Comelli EM, Arendt BM, Bonengel J, Fung SK, Fischer SE, McGilvray ID, Allard JP: Intestinal microbiota in patients with nonalcoholic fatty liver disease. Hepatology 2013;58:120-127.

35 Irrazábal T, Belcheva A, Girardin SE, Martin A, Philpott DJ: The multifaceted role of the intestinal microbiota in colon cancer. Mol Cell 2014;54:309-320.

36 Berer K, Mues M, Koutrolos M, Rasbi ZA, Boziki M, Johner C, Wekerle H, Krishnamoorthy G: Commensal microbiota and myelin autoantigen cooperate to trigger autoimmune demyelination. Nature 2011;479:538-541.

37 Koren O, Spor A, Felin J, Fåk F, Stombaugh J, Tremaroli V, Behre CJ, Knight R, Fagerberg B, Ley RE, Bäckhed F: Human oral, gut, and plaque microbiota in patients with atherosclerosis. Proc Natl Acad Sci U S A 2011;108(suppl 1):45924598.

38 Moayyedi P, Surette MG, Kim PT, Libertucci J, Wolfe M, Onischi C, Armstrong D, Marshall JK, Kassam Z, Reinisch W, Lee CH: Fecal microbiota transplantation induces remission in patients with active ulcerative colitis in a randomized controlled trial. Gastroenterology 2015;149:102-109.e6.

39 Rossen NG, Fuentes S, van der Spek, Mirjam J, Tijssen J, Hartman JHA, Duflou A, Löwenberg $\mathrm{M}$, van den Brink, Gijs R, Mathus-Vliegen EMH, de Vos WM, Zoetendal EG, D'Haens GR, Ponsioen CY: Findings from a randomized controlled trial of fecal transplantation for patients with ulcerative colitis. Gastroenterology 2015;149:110-118.e4.

40 Paramsothy S, Kamm MA, Kaakoush NO, Walsh AJ, van den Bogaerde J, Samuel D, Leong RWL, Connor S, Ng W, Paramsothy R, Xuan W, Lin E, Mitchell HM, Borody TJ: Multidonor intensive faecal microbiota transplantation for active ulcerative colitis: a randomised placebo-controlled trial. Lancet 2017; 389:1218-1228. 